DOI: https://doi.org/10.11144/Javeriana.umed61-3.inno

\title{
Innovación en las unidades de urgencias
}

Innovation in Emergency Units

Recepción: 31/08/2019 | Aceptación: 27/01/2020

\author{
Atilio Moreno-Carrillo ${ }^{a}$ \\ Médico internista. Advanced Fellowship in Emergency \\ Medicine. Magíster en Administración de Salud, \\ Colombia
}

a Autor de correspondencia: atilio.moreno@javeriana.edu.co

Cómo citar: Moreno-Carrillo A. Innovación en las unidades de urgencias. Univ. Med. 2020;61(3). https: //doi.org/10.11144/Javeriana.umed61-3.inno

\section{RESUMEN}

La humanidad siempre ha estado ligada a la innovación, y en su desarrollo el área de la salud no ha sido ajena a los beneficios de esta. En las últimas décadas se han evidenciado grandes avances y aportes en los procesos diagnósticos, terapéuticos y de rehabilitación de diversas enfermedades. La innovación en el sector de la salud debe ser un proceso de creación de valor para el individuo, la sociedad y las empresas, que mejore la eficiencia y genere beneficios sociales y económicos. Son múltiples los retos que se presentan día a día en el proceso de atención de las unidades de urgencias que podrían abordarse a través de soluciones innovadoras, por medio de la realización de acciones por fuera de los estándares tradicionales. Este artículo reflexiona en torno a la justificación y el propósito de innovar en las unidades de urgencias, y aborda estrategias y herramientas que se puedan consolidar y lograr que funcionen en áreas como el triaje, el modelo de atención de urgencias y las áreas de observación de urgencias, mencionando intervenciones planteadas por el International Emergency Department Leadership Institute y experiencias exitosas de la unidad de urgencias del Hospital Universitario San Ignacio.

Palabras clave

innovación; atención en urgencias; unidades de urgencias.

\begin{abstract}
Humanity has always been linked to innovation, and the health area has not been oblivious to the innovation benefits in its development. In recent decades there have been great advances and contributions in the diagnostic, therapeutic and rehabilitation processes of various diseases. Innovation in the health sector must be a process of creating value for the individual, society and companies, which improves efficiency and allows social and economic benefits to be generated. There are many challenges that arise day by day in the process of attention to the emergency units that could be addressed through innovative solutions, through the performance of actions outside the traditional standards. This article reflects on the justification and purpose of innovation in the emergency units, and addresses strategies and tools that can be used to consolidate and function in areas such as triage, emergency care model and areas of emergency observation, mentioning interventions raised by the International Emergency Department Leadership Institute (IEDLI) and successful experiences of the emergency unit of Hospital Universitario San Ignacio.

Keywords

innovation; emergency care; medical emergency services.
\end{abstract}




\section{Introducción}

La evolución de la humanidad siempre ha estado ligada a la innovación. Desde el fuego y la rueda, pasando por los sistemas económicos y de gobierno, hasta el automóvil, internet o la telefonía móvil, son múltiples las innovaciones que han marcado el curso de la historia.

En las últimas tres décadas, el tema de la innovación ha sido ampliamente analizado en diferentes sectores, y a esta se le considera el motor fundamental del desarrollo social y económico de los países.

El área de la salud no ha estado excluida de los beneficios de la innovación en su desarrollo. Por ejemplo, en el ámbito biotecnológico y farmacológico, se han evidenciado grandes avances y aportes en los procesos diagnósticos, terapéuticos y de rehabilitación de diversas enfermedades.

Debido a la relevancia de la innovación, es importante considerar su influencia en los procesos y en las actividades de las unidades de urgencias, teniendo en cuenta los múltiples retos que se presentan en estos servicios.

Este artículo reflexiona en torno a la justificación y el propósito de la innovación en las unidades de urgencias; además, proporciona ejemplos prácticos que permitan el desarrollo de estrategias y herramientas que se puedan utilizar para que esta se consolide y funcione en el modelo de atención de urgencias, y en diferentes áreas como el triaje y la observación de urgencias.

$\mathrm{Se}$ mencionan intervenciones planteadas por el International Emergency Department Leadership Institute (IEDLI), creado por la Facultad de Medicina de la Universidad de Harvard y otros expertos internacionales, y por experiencias exitosas de la unidad de urgencias del Hospital Universitario San Ignacio (HUSI), institución de salud de alta complejidad ubicada en Bogotá (Colombia), que en los últimos tres años (2016-2018) recibió en su unidad de urgencias 160.000 pacientes en promedio por año.

\section{Contexto del problema}

¿Por qué innovar? Desde hace más de una década, la innovación está presente en todas las agendas de desarrollo empresarial, incluida en la de la salud. En los servicios, la Organización para la Cooperación y el Desarrollo Económico (OCDE) y la Unión Europea la ubican en el portafolio de proyectos para la transformación social, haciendo hincapié en el usuario final, que en el caso de la atención en salud es el paciente y su familia (1).

Para el Foro Económico Mundial, la creatividad será la tercera habilidad más valorada por las empresas en el 2020 (2). De esta forma, la capacidad de innovación se constituye en una competencia imprescindible para enfrentar los retos que se presenten con el avance de las nuevas tecnologías.

Un estudio realizado por Price Water House \& Coopers encontró que el $20 \%$ de las empresas más innovadoras del mundo crecerá un $62 \%$ en los siguientes cinco años; mientras que el $20 \%$ de las menos innovadoras solo lo hará en un 20,7 \% (3).

En esta misma línea, cada año, el Índice Mundial de Innovación clasifica a cerca de 130 países de todo el mundo en función de sus resultados en materia de innovación, de acuerdo con 81 indicadores. En el 2017, ningún país suramericano ocupó uno los cinco primeros lugares de la lista, en la cual se ubicaron Suiza, Suecia, Países Bajos, Estados Unidos y Reino Unido. En América Latina y el Caribe, los países que mejor se situaron en el índice fueron Chile, Costa Rica y México (4).

Es motivo de preocupación que en el 2017, en ningún país de América Latina y el Caribe, los resultados en innovación superaran su grado de desarrollo; es más, en los últimos años, las clasificaciones del Índice Mundial de Innovación en la región no han mejorado significativamente con respecto a otras regiones. Así, el potencial de América Latina en innovación aún se encuentra por debajo de regiones como el sudeste asiático, Oceanía, el norte de África o Asia del este (4).

Lo anterior puede tener múltiples explicaciones; sin embargo, hay una que 
se destaca: mientras naciones altamente innovadoras como Corea del Sur o Israel invierten más del $4 \%$ de su producto interno bruto (PIB) en investigación y desarrollo de nuevos productos, y Suecia, Finlandia o Dinamarca invierten más del $3 \%$ de su PIB en estas mismas áreas, en Latinoamérica el promedio de inversión en innovación es del 0,5\%. De acuerdo con el Observatorio Colombiano de Ciencia y Tecnología, en el 2016 la inversión en innovación en nuestro país estuvo alrededor del $0,27 \%$ del PIB.

Ante este panorama, surge un importante interrogante: ipara qué innovar, dado que como lo afirmara Albert Einstein, "no podemos resolver nuestros problemas con el mismo pensamiento que usamos cuando los creamos"? En ese orden de ideas, la innovación en el sector de la salud debe ser un proceso de creación de valor para el individuo, la sociedad y las empresas. Esta creación de valor debe buscar la introducción de nuevos productos (bienes o servicios), procesos de producción, modelos de negocio y modelos organizativos para mejorar la eficiencia, la competitividad de las organizaciones, obtener beneficios sociales y generar beneficios económicos.

En la actualidad, son múltiples los retos que se presentan día a día en el proceso de atención de las unidades de urgencias que podrían ser abordados a través de soluciones innovadoras, por medio de la realización de acciones por fuera de los estándares tradicionales.

En este punto, es preciso distinguir entre generación de innovación y adopción de productos innovadores. Los procesos que generan innovación hacen que una idea se transforme en valor, de manera tal que la innovación debe proveer valor para el que la adquiere y para el innovador, incluyendo al generador de la idea y a las organizaciones que la desarrollaron, financiaron y gestionaron, con el objetivo de seguir generando desarrollos e impulsando la cultura de la innovación (5).

\section{Sobre la innovación}

La naturaleza de la innovación implica la idea de novedad, y sus efectos pueden manifestarse en un contexto micro, como la empresa, o en un contexto macro, como el mercado o incluso el mundo (6).

La innovación aporta a las organizaciones desarrollo en sus productos y servicios, pues se busca generar valor de forma diferenciada para el cliente respecto a otros competidores. Puede darse mediante cambios rápidos y disruptivos, pero también se presenta a través de cambios incrementales que generalmente se expresan bajo la forma de mejoras progresivas de productos, servicios o metodologías.

Para entrar en materia, se presentan dos definiciones sobre la innovación que se ajustan a las realidades de cualquier unidad de urgencias. En el Manual de Oslo, desarrollado en el 2005 por la OCDE, la innovación se entiende como la introducción de un nuevo o significativamente mejorado producto (bien o servicio), proceso, método de comercialización o método organizativo que busca mejorar u optimizar las prácticas internas de la empresa, la organización del lugar de trabajo y las relaciones exteriores (7).

Por otra parte, en su texto Economía naranja, el Banco Interamericano de Desarrollo definió la innovación como nuevas ideas que generan valor económico y social, una oportunidad de acelerar el proceso de mejorar vidas, repensando cómo generar soluciones alternativas, aprovechando la creatividad y la inteligencia colectiva (8).

\section{Los servicios de urgencias}

Antes de responder a la pregunta sobre cómo innovar en urgencias, vale la pena considerar algunas características propias de estos servicios. Las unidades de urgencias se caracterizan por ser áreas de alta complejidad asistencial y administrativa, no solo en cuanto a la atención de pacientes con enfermedades de alta complejidad médica o con múltiples morbilidades, sino también por el proceso de atención per se, el 
cual cuenta con gran cantidad de profesionales y tecnologías ampliamente diversas que entran en un complejo sistema de interacciones con el paciente y su familia. Esta atención se presta las veinticuatro horas del día, los siete días de la semana, y en nuestro medio, con frecuencia, se encuentra asociada a congestión y hacinamiento.

De acuerdo con la conceptualización de la cadena de valor y de los agentes del sector de la salud, realizada por Lawton R. Burns de la Escuela de Negocios de Wharton, existen cinco principales: 1) agentes reguladores, planificadores y financiadores, representados por la autoridad de salud; 2) agentes compradores de servicios de salud, representados por los servicios nacionales o regionales de salud y las compañías aseguradoras; 3) agentes proveedores de servicios, responsables de la prestación de servicios de salud, representados por los centros asistenciales; 4) agentes intermediarios de distribución de productos y servicios, representados por las plataformas logísticas y proveedores; y 5) agentes productores, representados por las empresas generadoras de productos farmacológicos y no farmacológicos $(9,10)$.

Si se considera lo anterior, para innovar en el sector de la salud se deberían fomentar y formular procesos innovadores en todos los agentes de este ámbito. Así, la autoridad correspondiente debería innovar en el modelo de salud; los agentes productores y agentes intermediarios, en la generación de nuevos productos y servicios farmacológicos y no farmacológicos, y las instituciones de salud, en la prestación del servicio.

\section{La innovación en urgencias}

En relación con los procesos de innovación en las unidades de urgencias, en primera instancia es importante vislumbrar algunos ejemplos que han partido de las recomendaciones del IEDLI, con el fin de proporcionar a los líderes de las unidades de urgencias habilidades y conocimientos para operar sus servicios de forma exitosa en cualquier lugar del mundo.
Para los servicios de urgencias, el IEDLI recomienda considerar un modelo de asistencia centrado en el paciente frente al modelo tradicional de atención secuencial. En este contexto, proponen la creación de unidades de asistencia rápida (UAR) a la entrada de los servicios de urgencias. En estas, los pacientes son evaluados de forma inmediata y simultáneamente, y no de forma secuencial. Todo tipo de paciente puede ser tratado en las UAR. Unos serán manejados y dados de alta desde la misma unidad, en tanto que otros comenzarán sus pruebas de diagnóstico y tratamiento para finalizar su proceso de atención en el área principal de urgencias. Es importante recalcar que en este modelo los pacientes críticos pasan inmediatamente al área de reanimación (11).

La implementación de estas unidades implica un cambio en el paradigma de atención en urgencias, porque significa el fin del triaje tal y como se conoce. La iniciativa de las UAR propone que el triaje se combine con la evaluación inicial del personal de enfermería y médico en el espacio que el paciente va a ocupar en el servicio de urgencias. Ello genera un acceso más rápido del paciente a la atención en urgencias, así como la posibilidad de acortar el tiempo total de la visita. Cierta evidencia sugiere que el acortamiento de estos tiempos de evaluación al inicio de la visita a urgencias beneficia la calidad asistencial ofrecida (12).

Por otra parte, el IEDLI también recomienda la implementación de un protocolo "código ayuda". En este escenario, cuando los pasillos de urgencias están colmados por camillas de pacientes esperando su ingreso, los pasillos de las áreas de hospitalización podrían ser ocupados por camillas de pacientes. En este contexto, también se deberían cancelar las cirugías y los procedimientos programados no urgentes y se deberían poner en marcha estrictos protocolos que verifiquen la duración de la hospitalización, basados en cuadros clínicos y medidas de calidad establecidas $(13,14)$.

Finalmente, el IEDLI plantea que se creen unidades de observación de urgencias (UOU), las cuales no deben considerarse unidades pequeñas de hospitalización, sino unidades efectivas de 
corta observación dentro del servicio. Estas áreas deben contar con protocolos médicos y de enfermería bien definidos para los pacientes que ingresan en ellas, así como límites en los tiempos de estancia. Cuando se supere ese límite de tiempo de estancia, los pacientes de las UOU tendrían prioridad de hospitalización. En términos generales, las UOU no deberían usarse como unidades de espera de hospitalización, pero si lo llegan a ser, los equipos médicos y de enfermería de las unidades de hospitalización deberían hacerse cargo de los pacientes en las UOU (15).

\section{El caso del Hospital Universitario San Ignacio}

Para continuar con experiencias de innovación en la prestación de servicio en unidades de urgencias, se refiere la experiencia de la unidad de urgencias del HUSI, institución de salud de cuarto nivel de atención ubicada en Bogotá, Colombia.

En el 2017 se decidió trasladar la responsabilidad de cuidado de los pacientes del área de observación de urgencias al grupo médico y de enfermería del área de hospitalización. Lo anterior, teniendo en cuenta que la observación de urgencias se había convertido en un área de espera de traslado a las áreas de hospitalización de pacientes con orden de internación. De esta forma, la atención de los pacientes con patologías médicas y quirúrgicas con orden de manejo intrahospitalario se delegó a las áreas de hospitalización responsables (grupo médico y de enfermería), y en este espacio se instauraron todos los protocolos de manejo de hospitalización.

Con esta medida, y con el seguimiento de un gestor del área, en el tercer trimestre del 2017 se logró aumentar los egresos efectivos del área en diez puntos porcentuales, en tanto que la rotación de pacientes con patologías infecciosas creció en un $15 \%$.

En otro proceso innovador que se realizó en la unidad de urgencias del HUSI, se evaluó el tiempo trascurrido entre la llegada del paciente al servicio y la realización del triaje (pretriaje), y se aplicó la teoría de colas. En un primer periodo de evaluación de 7 meses, comprendido entre agosto y febrero de los años 2016-2017, se consideraron 89.898 turnos, con un tiempo promedio pretriaje de 22,15 minutos. De acuerdo con la distribución de llegada de los pacientes, y considerando los resultados de los cálculos obtenidos utilizando la teoría de colas, se determinó el número de enfermeros necesarios en el servicio para la realización del triaje por hora, para cada día de la semana, y se implementaron los cambios necesarios sin aumentar el personal contratado. Para un segundo periodo de evaluación de también 7 meses, trascurridos entre agosto y febrero de los años 2017-2018, se consideraron 94.497 turnos, que demostraron una reducción del tiempo promedio pretriaje a 7,5 minutos. De esta forma, la planeación utilizando la teoría de colas redujo en un $65 \%$ el tiempo pretriaje sin incurrir en costos adicionales (16).

\section{¿Cómo hacer que la innovación funcione en las unidades de urgencias?}

Es claro que la innovación que aporta un mayor valor diferenciador es la que se origina en la convicción de los líderes de las organizaciones: es necesario cuestionar los factores sobre los que se basa la competitividad organizacional y la forma de gestionarlos. Por lo tanto, es fundamental contar con el pleno respaldo y el compromiso de los directores institucionales para que cualquier proceso innovador funcione (17).

Generar espacios de innovación implica un gran reto para los responsables de las áreas de urgencias y los directores institucionales. Por ello, establecerlos podría ser el primer paso hacia un proceso sólido de generación de innovación.

El apoyo a la innovación suele generar más innovación. Por consiguiente, los líderes que respaldan abiertamente la innovación pueden inspirar ideas creativas entre sus empleados (18). Una encuesta enviada por la Sociedad Americana de Cirugía preguntó a los especialistas de esa área sobre las dificultades para investigar. Se obtuvieron 1033 respuestas (tasa de respuesta del $41 \%$ ), donde el $66 \%$ de los que respondieron 
indicó que la falta de tiempo y la conciliación entre trabajo y vida privada fueron las barreras más importantes (17).

En relación con lo anterior, es fundamental institucionalizar y formalizar la innovación en el hospital y sus diferentes departamentos y unidades, a fin de garantizar con ello una dedicación específica y especializada. En ese sentido, una acción muy importante en el desarrollo e impulso de la innovación en las unidades de urgencias es que esta cuente como una actividad más del grupo técnico y profesional y que, de esta manera, genere tiempos determinados para innovar dentro de los horarios laborales. Garantizar estos espacios protegidos e idealmente multidisciplinarios, de acuerdo con las características de cada unidad de urgencias y el personal que la conforma, puede generar un ambiente donde los empleados reflexionen sobre las características del proceso de atención y se favorezca, a través de grupos de innovación, la generación de actividades que produzcan valor. En este contexto, se debe asegurar que los colaboradores trabajen en los procesos de innovación en equipo, que se les remunere por su participación y que esta actividad no afecte la atención de los pacientes (19).

Por otra parte, a partir de las fortalezas propias de la multidisciplinariedad de las unidades de urgencias, los procesos de innovación deberían contar con creatividad artística, la cual involucra imaginación y capacidad para originar ideas originales y novedosas; creatividad científica, que se activa desde la curiosidad por la observación de fenómenos y la disposición para experimentar y crear nuevas formas para resolver los problemas; y creatividad económica, que apunta a la innovación en tecnologías de producción, prácticas comerciales y mercadeo (8). Si una unidad de urgencias desea que sus procesos de innovación funcionen, debe garantizar que sus grupos de innovación cuenten con personas con las competencias previamente descritas.

Según el Boston Consulting Group, existen cuatro claves que impulsan la innovación: la generación de ideas, los procesos estructurados, el liderazgo y los empleados capacitados, y para ellos este último factor es el más importante para los procesos de innovación en una empresa (20).

Encontrar empleados o colaboradores con el conocimiento y las habilidades necesarias para promover la innovación puede resultar un desafío, y en este aspecto cobran gran importancia la capacitación de los empleados interesados en innovación, así como la búsqueda activa de quienes cuenten con las competencias para innovar.

Aunque los líderes de las unidades de urgencias deben concentrarse en la búsqueda de talentos naturales entre el personal de sus servicios y permitirles que pongan en práctica sus fortalezas individuales, también deben generar espacios de capacitación sobre: procesos de innovación en urgencias, grupos de generación de ideas, liderazgo, técnicas eficientes de creación de valor en urgencias, propiedad intelectual, patentes, evaluación y validación de conceptos o dispositivos y atributos de una cultura de innovación organizacional sostenible, por citar algunos ejemplos.

Este tipo de capacitaciones se pueden brindar en sesiones cortas presenciales o a través de módulos de enseñanza virtual. En el caso de que la organización no cuente con expertos internos que puedan brindar capacitación sobre innovación, es recomendable contar con asesores externos que se encarguen de esta actividad.

Adicionalmente a lo señalado hasta aquí, para desarrollar y consolidar los procesos de innovación en las unidades de urgencias, los líderes deben asumir la responsabilidad de generar apoyo eficaz y sistemas de recompensas que motiven a los empleados a transformarse en innovadores. Estas recompensas, habituales en otros sectores, se pueden dar a través de retribuciones monetarias (21). Sin embargo, en el contexto de los servicios de urgencias es importante la flexibilidad.

$\mathrm{Si}$ se tiene en cuenta que lo que motiva a las personas en un entorno y en un momento determinado puede no ser igual a lo que las motiva en otros entornos o momentos, es posible que en muchas circunstancias los miembros de los grupos de innovación prefieran compensaciones que no sean monetarias (22). 
Los líderes de las unidades de urgencias deben conocer muy bien a sus grupos y establecer con antelación, y de forma concertada, la mejor recompensa de los procesos innovadores, la cual puede ser con bonificaciones en tiempo, en periodos de descanso, con trabajo en casa, en apoyo formativo o en dinero.

Finalmente, la cultura de la innovación debe trascender el propio hospital y fortalecerse a través de la construcción de redes interdisciplinarias y grupos de interés. En este sentido, resulta fundamental la participación de universidades y sociedades científicas, así como el financiamiento del sector público y privado.

En este aspecto se debe considerar la colaboración de estadísticos, ingenieros, creadores de software y líderes empresariales, para que aporten a los procesos de innovación de las unidades de urgencias los conocimientos y las lecciones aprendidas en otras disciplinas, que usualmente desconoce la mayoría del personal de la salud. Es claro que por medio del trabajo interdisciplinario se pueden obtener mejores y mayores logros, a través de la integración de datos, las múltiples perspectivas y los conceptos de diferentes disciplinas.

En la actualidad, existen ejemplos de trabajo colaborativo que vale la pena emular, como los de Medintech (23), en España, o el National Innovation Center (24), en el Reino Unido, espacios virtuales que facilitan la interacción entre instituciones públicas y privadas que están innovando o buscando soluciones innovadoras.

\section{Conclusiones}

Los líderes de las unidades de urgencias pueden encontrar en la innovación una estrategia efectiva para solucionar sus problemas. Aspectos como los retrasos en la atención, la congestión, el hacinamiento o los egresos de usuarios sin valoración médica son situaciones que deberían abordarse por medio de procesos innovadores.

Favorecer la creación de UAR, donde los pacientes son evaluados de forma inmediata y simultáneamente, y el triaje se combina con la evaluación inicial del paciente por parte del personal de enfermería y médico; la implementación del protocolo "código ayuda", que facilita el traslado de los pacientes de los pasillos de urgencias a los pasillos de las áreas de hospitalización (en casos de extrema congestión); y la creación de UOU, con protocolos médicos y de enfermería bien definidos y con límites en los tiempos de estancia, son estrategias innovadoras planteadas por el IEDLI.

Las experiencias exitosas de innovación en el HUSI, relacionadas con trasladar la responsabilidad del cuidado de los pacientes del área de observación de urgencias al grupo médico y de enfermería del área de hospitalización, y la aplicación de la teoría de colas en el proceso de triaje, para establecer el número de enfermeros necesarios para la realización de esta actividad, generaron en la unidad de urgencias un aumento en los egresos efectivos del área de observación en diez puntos porcentuales y una reducción significativa del tiempo promedio de pretriaje.

Estos ejemplos de innovación podrían ser adoptados o adaptados de acuerdo con las características propias de otras unidades de urgencias, lo que generaría eficiencias en los procesos asistenciales o administrativos.

La creación en los servicios de urgencias de grupos multidisciplinarios, encargados de desarrollar pensamiento creativo e innovador, es fundamental para cambiar el quehacer habitual y fortalecer la cultura de innovación.

Entre más personas clave (con competencias y motivación) participen en los procesos de innovación en las unidades de urgencias, más sencillo será el desarrollo de los proyectos y la implementación de los resultados alcanzados. Por lo tanto, el fomento, el apoyo y el estímulo de grupos colaborativos multi e interdisciplinarios son de vital importancia para el logro de estos objetivos.

Es fundamental garantizar que la innovación en los servicios de urgencias cuente como una actividad más del grupo asistencial y que se generen tiempos protegidos dentro de los horarios laborales para su desarrollo. También es prioritario que en el proceso de consolidación de la innovación en las unidades de urgencias se dé la participación de las universidades y sociedades 
científicas y el financiamiento del sector público y privado.

Conflictos de interés: el autor no tiene conflictos de interés.

Financiación: el autor.

\section{Referencias}

1. European Commission. Communication from the Commission Europe 2020: A strategy for smart, sustainable and inclusive growth [internet]. Bruselas; 2010. Disponible en: http://eurlex.europa.eu/LexUriSer v/LexUriServ.do?uri=COM:2010:202 0:FIN:EN:PDF

2. World Economic Forum. The future of jobs employment, skills and workforce strategy for the fourth industrial revolution [internet]; 2016. Disponible en: http://www3.weforum.o rg/docs/WEF_Future_of_Jobs.pdf

3. Shelton R, Percival D. Breakthrough innovation and growth [internet]. Price Water House Coopers; 2013. Disponible en: https://www.pwc.es/es/publicacion es/gestion-empresarial/assets/breakthr ough-innovation-growth.pdf

4. Cornell University, INSEAD, WIPO. The Global Innovation Index 2017: Innovation feeding the world [internet]; 2017. Disponible en: https:/www.wipo.int/publications/ es/details.jsp?id=4193

5. Blanch L, Annane D, Antonell M, Chiche JD, Cunat J, Girard TD, et al. The future of intensive care medicine. Med Intensiva. 2013;37:91-8.

6. Spiezia V. Are ICT users more innovative?: an analysis of ICT-enabled innovation in OECD firms. OECD Journal Economic Studies [internet]. 2011;2011(1):1-21. https://doi.org/10. 1787/eco_studies-2011-5kg2d2hkn6vg
7. Organización para la Cooperación y el Desarrollo Económico. Medición de las actividades científicas y tecnológicas: directrices propuestas para recabar e interpretar datos de la innovación tecnológica. Manual Oslo. París: OCDE; 2005.

8. Banco Interamericano de Desarrollo. Economía naranja: innovaciones que no sabías que eran de América Latina y el Caribe. Washington: BID; 2017.

9. Burns LR. The health care value chain: producers, purchasers, and providers. San Francisco: Jossey-Bass; 2002.

10. Burns LR. The business of health care innovation. Cambridge: Cambridge University Press; 2012.

11. Sayah A, Lai-Becker M, Kingsley-Rocker L, Scott-Long T, O'Connor K, Lobón LF. Emergency department expansion versus patient flow improvement: impact on patient experience of care. J Emerg Med. 2016;50:339-48.

12. Improved flow aids patient safety. ED Manag. 2011;23(1):10-1.

13. Executive Office of Health and Human Services. The Commonwealth of Massachusetts Executive Office of Health and Human Services Department of Public Health Bureau of Health Care Safety and Quality, 2015 [internet]. Disponible en: http://www.mass.gov/eohhs/docs/d $\mathrm{ph} /$ quality/healthcare/codehelp/code-h elp-update-060315.pdf

14. Khazaeni B, Novales J, Voigt J, Gaffud G, Le J, Stillings J, Mouri M. The surge plan: improving emergency department overcrowding [internet]; 2018 Mar 26. Disponible en: https://www.vituity.com/blog/the-s urge-plan-improving-emergency-depar tment-overcrowding/ 
15. Salvador-Kelly AV, Nancy S, Kwon NS. Emergency department observation units. Emerg Med Rep. 2016;April 15.

16. Moreno-Carrillo A, Arenas LM, Fonseca JA, Caicedo CA, Tovar SV, Muñoz-Velandia O. Application of queuing theory to optimize the Triage process in a tertiary emergency care ("ER") department. J Emerg Trauma Shock. 2019;12:268-73.

17. Keswani SG, Moles CM, Morowitz M, Zeh H, Kuo JS, Levine MH, et al. Basic Science Committee of the Society of University Surgeons. The future of basic science in academic surgery: Identifying barriers to success for surgeon-scientists. Ann Surg. 2016;16:1.

18. Eisenbeiss SA, van Knippenberg D, Boerner S. Transformational leadership and team innovation: integrating team climate principles. J Appl Psychol. 2008 Nov;93(6):1438-46.

19. Wilson J, Speroni KG, Jones RA, Daniel MG. Exploring how nurses and managers perceive shared governance. Nursing. 2014 Jul;44(7):19-22.

20. Andrew JP, Stover DeRocco E, Taylor A. Innovation imperative in manufacturing: How the United States can restore its edge [internet]. Boston: The Boston Consulting Group; 2009. Disponible en: http://www.themanufacturinginstit ute.org/ /media/6731673D21A64259 B081AC8E083AE091.ashx

21. The World Bank. World development report: Gender equality and development [internet]. Washington; 2012. Disponible en: https://siteresources.worldbank.org /INTWDR2012/Resources/7778105-1 299699968583/7786210-13159362220

06/Complete-Report.pdf
22. Glor ED. Innovation patterns. Innovation Journal [internet]. 2001;6(3). Disponible en: http://www.innovation.cc/volumes -issues/vol6-iss3.htm

23. Medintech [Internet]. Disponible en: http://www.medintech.es/home.as px

24. National Innovation Centre. Knowledge and information zone [Internet]. Disponible en: http://knowl edge.nic.nhs.uk. 